\title{
INEQUALITIES FOR HYPO- $q$-NORMS ON A CARTESIAN PRODUCT OF INNER PRODUCT SPACES
}

\author{
SILVESTRU SEVER DRAGOMIR ${ }^{1,2}$
}

\begin{abstract}
In this paper we introduce the hypo- $q$-norms on a Cartesian product of inner product spaces. A representation of these norms in terms of inner products, the equivalence with the $q$-norms on a Cartesian product and some reverse inequalities obtained via the scalar Shisha-Mond, Birnacki et al., Grüss type inequalities, Boas-Bellman and Bombieri type inequalities are also given.
\end{abstract}

\section{INTRODUCTION}

Let $(E,\|\cdot\|)$ be a normed linear space over the real or complex number field $\mathbb{K}$. On $\mathbb{K}^{n}$ endowed with the canonical linear structure we consider a norm $\|\cdot\|_{n}$ and the unit ball

$$
\mathbb{B}\left(\|\cdot\|_{n}\right):=\left\{\boldsymbol{\lambda}=\left(\lambda_{1}, \ldots, \lambda_{n}\right) \in \mathbb{K}^{n} \mid\|\lambda\|_{n} \leq 1\right\} .
$$

As an example of such norms we should mention the usual p-norms

$$
\|\boldsymbol{\lambda}\|_{n, p}:= \begin{cases}\max \left\{\left|\lambda_{1}\right|, \ldots,\left|\lambda_{n}\right|\right\} & \text { if } p=\infty ; \\ \left(\sum_{k=1}^{n}\left|\lambda_{k}\right|^{p}\right)^{\frac{1}{p}} & \text { if } p \in[1, \infty) .\end{cases}
$$

The Euclidean norm is obtained for $p=2$, i.e.,

$$
\|\boldsymbol{\lambda}\|_{n, 2}=\left(\sum_{k=1}^{n}\left|\lambda_{k}\right|^{2}\right)^{\frac{1}{2}}
$$

It is well known that on $E^{n}:=E \times \cdots \times E$ endowed with the canonical linear structure we can define the following $p$-norms:

$$
\|\mathbf{x}\|_{n, p}:= \begin{cases}\max \left\{\left\|x_{1}\right\|, \ldots,\left\|x_{n}\right\|\right\} & \text { if } p=\infty ; \\ \left(\sum_{k=1}^{n}\left\|x_{k}\right\|^{p}\right)^{\frac{1}{p}} & \text { if } p \in[1, \infty) ;\end{cases}
$$

where $\mathbf{x}=\left(x_{1}, \ldots, x_{n}\right) \in E^{n}$.

Following [9], for a given norm $\|\cdot\|_{n}$ on $\mathbb{K}^{n}$, we define the functional $\|\cdot\|_{h, n}$ : $E^{n} \rightarrow[0, \infty)$ given by

$$
\|\mathbf{x}\|_{h, n}:=\sup _{\lambda \in B\left(\|\cdot\|_{n}\right)}\left\|\sum_{j=1}^{n} \lambda_{j} x_{j}\right\|,
$$

where $\mathbf{x}=\left(x_{1}, \ldots, x_{n}\right) \in E^{n}$.

1991 Mathematics Subject Classification. 46C05; 26D15.

Key words and phrases. Normed spaces, Cartesian products of normed spaces, Inequalities, Reverse inequalities, Shisha-Mond, Birnacki inequality, Grüss type inequalities, Boas-Bellman and Bombieri type inequalities. 
It is easy to see, by the properties of the norm $\|\cdot\|$, that:

(i) $\|\mathbf{x}\|_{h, n} \geq 0$ for any $\mathbf{x} \in E^{n}$;

(ii) $\|\mathbf{x}+\mathbf{y}\|_{h, n} \leq\|\mathbf{x}\|_{h, n}+\|\mathbf{y}\|_{h, n}$ for any $\mathbf{x}, \mathbf{y} \in E^{n}$;

(iii) $\|\alpha \mathbf{x}\|_{h, n}=|\alpha|\|\mathbf{x}\|_{h, n}$ for each $\alpha \in \mathbb{K}$ and $\mathbf{x} \in E^{n}$;

and therefore $\|\cdot\|_{h, n}$ is a semi-norm on $E^{n}$. This will be called the hypo-seminorm generated by the norm $\|\cdot\|_{n}$ on $E^{n}$.

We observe that $\|\mathbf{x}\|_{h, n}=0$ if and only if $\sum_{j=1}^{n} \lambda_{j} x_{j}=0$ for any $\left(\lambda_{1}, \ldots, \lambda_{n}\right) \in$ $B\left(\|\cdot\|_{n}\right)$. If there exists $\lambda_{1}^{0}, \ldots, \lambda_{n}^{0} \neq 0$ such that $\left(\lambda_{1}^{0}, 0, \ldots, 0\right),\left(0, \lambda_{2}^{0}, \ldots, 0\right), \ldots$, $\left(0,0, \ldots, \lambda_{n}^{0}\right) \in B\left(\|\cdot\|_{n}\right)$ then the semi-norm generated by $\|\cdot\|_{n}$ is a norm on $E^{n}$.

If by $\mathbb{B}_{n, p}$ with $p \in[1, \infty]$ we denote the balls generated by the $p$-norms $\|\cdot\|_{n, p}$ on $\mathbb{K}^{n}$, then we can obtain the following hypo-q-norms on $E^{n}$ :

$$
\|\mathbf{x}\|_{h, n, q}:=\sup _{\boldsymbol{\lambda} \in \mathbb{B}_{n, p}}\left\|\sum_{j=1}^{n} \lambda_{j} x_{j}\right\|,
$$

with $q>1$ and $\frac{1}{q}+\frac{1}{p}=1$ if $p>1, q=1$ if $p=\infty$ and $q=\infty$ if $p=1$.

For $p=2$, we have the Euclidean ball in $\mathbb{K}^{n}$, which we denote by $\mathbb{B}_{n}, \mathbb{B}_{n}=$ $\left\{\boldsymbol{\lambda}=\left.\left(\lambda_{1}, \ldots, \lambda_{n}\right) \in \mathbb{K}^{n}\left|\sum_{i=1}^{n}\right| \lambda_{i}\right|^{2} \leq 1\right\}$ that generates the hypo-Euclidean norm on $E^{n}$, i.e.,

$$
\|\mathbf{x}\|_{h, e}:=\sup _{\boldsymbol{\lambda} \in \mathbb{B}_{n}}\left\|\sum_{j=1}^{n} \lambda_{j} x_{j}\right\| .
$$

Moreover, if $E=H, H$ is a inner product space over $\mathbb{K}$, then the hypo-Euclidean norm on $H^{n}$ will be denoted simply by

$$
\|\mathbf{x}\|_{e}:=\sup _{\boldsymbol{\lambda} \in \mathbb{B}_{n}}\left\|\sum_{j=1}^{n} \lambda_{j} x_{j}\right\| .
$$

Let $(H ;\langle\cdot, \cdot\rangle)$ be a Hilbert space over $\mathbb{K}$ and $n \in \mathbb{N}, n \geq 1$. In the Cartesian product $H^{n}:=H \times \cdots \times H$, for the $n$-tuples of vectors $\mathbf{x}=\left(x_{1}, \ldots, x_{n}\right), \mathbf{y}=$ $\left(y_{1}, \ldots, y_{n}\right) \in H^{n}$, we can define the inner product $\langle\cdot, \cdot\rangle$ by

$$
\langle\mathbf{x}, \mathbf{y}\rangle:=\sum_{j=1}^{n}\left\langle x_{j}, y_{j}\right\rangle, \quad \mathbf{x}, \mathbf{y} \in H^{n},
$$

which generates the Euclidean norm $\|\cdot\|_{2}$ on $H^{n}$, i.e.,

$$
\|\mathbf{x}\|_{2}:=\left(\sum_{j=1}^{n}\left\|x_{j}\right\|^{2}\right)^{\frac{1}{2}}, \quad \mathbf{x} \in H^{n} .
$$

The following result established in [9] connects the usual Euclidean norm $\|\cdot\|$ with the hypo-Euclidean norm $\|\cdot\|_{e}$.

Theorem 1 (Dragomir, 2007, [9]). For any $\mathbf{x} \in H^{n}$ we have the inequalities

$$
\frac{1}{\sqrt{n}}\|\mathbf{x}\| \leq\|\mathbf{x}\|_{e} \leq\|\mathbf{x}\|_{2}
$$

i.e., $\|\cdot\|_{2}$ and $\|\cdot\|_{e}$ are equivalent norms on $H^{n}$. 
The following representation result for the hypo-Euclidean norm plays a key role in obtaining various bounds for this norm:

Theorem 2 (Dragomir, 2007, [9]). For any $\mathbf{x} \in H^{n}$ with $\mathbf{x}=\left(x_{1}, \ldots, x_{n}\right)$, we have

$$
\|\mathbf{x}\|_{e}=\sup _{\|y\| \leq 1}\left(\sum_{j=1}^{n}\left|\left\langle x_{j}, y\right\rangle\right|^{2}\right)^{\frac{1}{2}} .
$$

Motivated by the above results, in this paper we introduce the hypo- $q$-norms on a Cartesian product of inner product spaces. A representation of these norms in terms of inner products, the equivalence with the $q$-norms on a Cartesian product and some reverse inequalities obtained via the scalar Shisha-Mond, Birnacki et al. and other Grüss type inequalities are also given.

\section{General Results}

Let $(H,\langle\cdot, \cdot\rangle)$ be an inner product space over the real or complex number field $\mathbb{K}$. We have the following representation result for the hypo-q-norms on $H^{n}$.

Theorem 3. Let $(H,\langle\cdot, \cdot\rangle)$ be an inner product space over the real or complex number field $\mathbb{K}$. For any $\mathbf{x} \in H^{n}$ with $\mathbf{x}=\left(x_{1}, \ldots, x_{n}\right)$, we have

$$
\|\mathbf{x}\|_{h, n, q}=\sup _{\|y\| \leq 1}\left\{\left(\sum_{j=1}^{n}\left|\left\langle x_{j}, y\right\rangle\right|^{q}\right)^{1 / q}\right\}
$$

where $p, q>1$ with $\frac{1}{p}+\frac{1}{q}=1$,

$$
\|\mathbf{x}\|_{h, n, 1}=\sup _{\|y\| \leq 1}\left\{\sum_{j=1}^{n}\left|\left\langle x_{j}, y\right\rangle\right|\right\}
$$

and

$$
\|\mathbf{x}\|_{h, n, \infty}=\|\mathbf{x}\|_{n, \infty}=\max _{j \in\{1, \ldots, n\}}\left\{\left\|x_{j}\right\|\right\}
$$

In particular,

$$
\|\mathbf{x}\|_{h, e}=\sup _{\|y\| \leq 1}\left\{\left(\sum_{j=1}^{n}\left|\left\langle x_{j}, y\right\rangle\right|^{2}\right)^{1 / 2}\right\} .
$$

Proof. Using Hölder's discrete inequality for $p, q>1$ and $\frac{1}{p}+\frac{1}{q}=1$ we have

$$
\left|\sum_{j=1}^{n} \alpha_{j} \beta_{j}\right| \leq\left(\sum_{j=1}^{n}\left|\alpha_{j}\right|^{p}\right)^{1 / p}\left(\sum_{j=1}^{n}\left|\beta_{j}\right|^{q}\right)^{1 / q},
$$

which implies that

$$
\sup _{\|\alpha\|_{p} \leq 1}\left|\sum_{j=1}^{n} \alpha_{j} \beta_{j}\right| \leq\|\beta\|_{q}
$$

where $\alpha=\left(\alpha_{1}, \ldots, \alpha_{n}\right)$ and $\beta=\left(\beta_{1}, \ldots, \beta_{n}\right)$. 
For $\left(\beta_{1}, \ldots, \beta_{n}\right) \neq 0$, consider $\alpha=\left(\alpha_{1}, \ldots, \alpha_{n}\right)$ with

$$
\alpha_{j}:=\frac{\overline{\beta_{j}}\left|\beta_{j}\right|^{q-2}}{\left(\sum_{k=1}^{n}\left|\beta_{k}\right|^{q}\right)^{1 / p}}
$$

for those $j$ for which $\beta_{j} \neq 0$ and $\alpha_{j}=0$, for the rest.

We observe that

$$
\begin{aligned}
\left|\sum_{j=1}^{n} \alpha_{j} \beta_{j}\right| & =\left|\sum_{j=1}^{n} \frac{\overline{\beta_{j}}\left|\beta_{j}\right|^{q-2}}{\left(\sum_{k=1}^{n}\left|\beta_{k}\right|^{q}\right)^{1 / p}} \beta_{j}\right|=\frac{\sum_{j=1}^{n}\left|\beta_{j}\right|^{q}}{\left(\sum_{k=1}^{n}\left|\beta_{k}\right|^{q}\right)^{1 / p}} \\
& =\left(\sum_{j=1}^{n}\left|\beta_{j}\right|^{q}\right)^{1 / q}=\|\beta\|_{q}
\end{aligned}
$$

and

$$
\begin{aligned}
\|\alpha\|_{p}^{p} & =\sum_{j=1}^{n}\left|\alpha_{j}\right|^{p}=\sum_{j=1}^{n} \frac{\left.\left.\left|\overline{\beta_{j}}\right| \beta_{j}\right|^{q-2}\right|^{p}}{\left(\sum_{k=1}^{n}\left|\beta_{k}\right|^{q}\right)}=\sum_{j=1}^{n} \frac{\left(\left|\beta_{j}\right|^{q-1}\right)^{p}}{\left(\sum_{k=1}^{n}\left|\beta_{k}\right|^{q}\right)} \\
& =\sum_{j=1}^{n} \frac{\left|\beta_{j}\right|^{q p-p}}{\left(\sum_{k=1}^{n}\left|\beta_{k}\right|^{q}\right)}=\sum_{j=1}^{n} \frac{\left|\beta_{j}\right|^{q}}{\left(\sum_{k=1}^{n}\left|\beta_{k}\right|^{q}\right)}=1 .
\end{aligned}
$$

Therefore, by (2.5) we have the representation

$$
\sup _{\|\alpha\|_{p} \leq 1}\left|\sum_{j=1}^{n} \alpha_{j} \beta_{j}\right|=\|\beta\|_{q}
$$

for any $\beta=\left(\beta_{1}, \ldots, \beta_{n}\right) \in \mathbb{K}^{n}$.

By the properties of inner product, we have for any $u \in H, u \neq 0$ that

$$
\|u\|=\sup _{\|y\| \leq 1}|\langle u, y\rangle| \text {. }
$$

Let $\alpha=\left(\alpha_{1}, \ldots, \alpha_{n}\right) \in \mathbb{K}^{n}$ and $\mathbf{x} \in H^{n}$ with $\mathbf{x}=\left(x_{1}, \ldots, x_{n}\right)$. Then by $(2.7)$ we have

$$
\left\|\sum_{j=1}^{n} \alpha_{j} x_{j}\right\|=\sup _{\|y\| \leq 1}\left|\left\langle\sum_{j=1}^{n} \alpha_{j} x_{j}, y\right\rangle\right|=\sup _{\|y\| \leq 1}\left|\sum_{j=1}^{n} \alpha_{j}\left\langle x_{j}, y\right\rangle\right| .
$$

By taking the supremum in this equality we have

$$
\begin{aligned}
\sup _{\|\alpha\|_{p} \leq 1}\left\|\sum_{j=1}^{n} \alpha_{j} x_{j}\right\| & =\sup _{\|\alpha\|_{p} \leq 1}\left(\sup _{\|y\| \leq 1}\left|\sum_{j=1}^{n} \alpha_{j}\left\langle x_{j}, y\right\rangle\right|\right) \\
& =\sup _{\|y\| \leq 1}\left(\sup _{\|\alpha\|_{p} \leq 1}\left|\sum_{j=1}^{n} \alpha_{j}\left\langle x_{j}, y\right\rangle\right|\right)=\sup _{\|y\| \leq 1}\left(\sum_{j=1}^{n}\left|\left\langle x_{j}, y\right\rangle\right|^{q}\right)^{1 / 2},
\end{aligned}
$$

where for the last equality we used the representation (2.6).

This proves (2.1). 
Using the properties of the modulus, we have

$$
\left|\sum_{j=1}^{n} \alpha_{j} \beta_{j}\right| \leq \max _{j \in\{1, \ldots, n\}}\left|\alpha_{j}\right| \sum_{j=1}^{n}\left|\beta_{j}\right|,
$$

which implies that

$$
\sup _{\|\alpha\|_{\infty} \leq 1}\left|\sum_{j=1}^{n} \alpha_{j} \beta_{j}\right| \leq\|\beta\|_{1},
$$

where $\alpha=\left(\alpha_{1}, \ldots, \alpha_{n}\right)$ and $\beta=\left(\beta_{1}, \ldots, \beta_{n}\right)$.

For $\left(\beta_{1}, \ldots, \beta_{n}\right) \neq 0$, consider $\alpha=\left(\alpha_{1}, \ldots, \alpha_{n}\right)$ with $\alpha_{j}:=\frac{\overline{\beta_{j}}}{\left|\beta_{j}\right|}$ for those $j$ for which $\beta_{j} \neq 0$ and $\alpha_{j}=0$, for the rest.

We have

$$
\left|\sum_{j=1}^{n} \alpha_{j} \beta_{j}\right|=\left|\sum_{j=1}^{n} \frac{\overline{\beta_{j}}}{\left|\beta_{j}\right|} \beta_{j}\right|=\sum_{j=1}^{n}\left|\beta_{j}\right|=\|\beta\|_{1}
$$

and

$$
\|\alpha\|_{\infty}=\max _{j \in\{1, \ldots, n\}}\left|\alpha_{j}\right|=\max _{j \in\{1, \ldots, n\}}\left|\frac{\overline{\beta_{j}}}{\left|\beta_{j}\right|}\right|=1
$$

and by (2.9) we get the representation

$$
\sup _{\|\alpha\|_{\infty} \leq 1}\left|\sum_{j=1}^{n} \alpha_{j} \beta_{j}\right|=\|\beta\|_{1}
$$

for any $\beta=\left(\beta_{1}, \ldots, \beta_{n}\right) \in \mathbb{K}^{n}$.

By taking the supremum in the equality (2.8) we have

$$
\begin{aligned}
\sup _{\|\alpha\|_{\infty} \leq 1}\left\|\sum_{j=1}^{n} \alpha_{j} x_{j}\right\| & =\sup _{\|\alpha\|_{\infty} \leq 1}\left(\sup _{\|y\| \leq 1}\left|\sum_{j=1}^{n} \alpha_{j}\left\langle x_{j}, y\right\rangle\right|\right) \\
& =\sup _{\|f\| \leq 1}\left(\sup _{\|\alpha\|_{\infty} \leq 1}\left|\sum_{j=1}^{n} \alpha_{j}\left\langle x_{j}, y\right\rangle\right|\right)=\sup _{\|f\| \leq 1}\left(\sum_{j=1}^{n}\left|\left\langle x_{j}, y\right\rangle\right|\right),
\end{aligned}
$$

where for the last equality we used the equality (2.10), which proves the representation (2.2).

Finally, we have

$$
\left|\sum_{j=1}^{n} \alpha_{j} \beta_{j}\right| \leq \sum_{j=1}^{n}\left|\alpha_{j}\right| \max _{j \in\{1, \ldots, n\}}\left|\beta_{j}\right|,
$$

which implies that

$$
\sup _{\|\alpha\|_{1} \leq 1}\left|\sum_{j=1}^{n} \alpha_{j} \beta_{j}\right| \leq\|\beta\|_{\infty},
$$

where $\alpha=\left(\alpha_{1}, \ldots, \alpha_{n}\right)$ and $\beta=\left(\beta_{1}, \ldots, \beta_{n}\right)$. 
For $\left(\beta_{1}, \ldots, \beta_{n}\right) \neq 0$, let $j_{0} \in\{1, \ldots, n\}$ such that $\|\beta\|_{\infty}=\max _{j \in\{1, \ldots, n\}}\left|\beta_{j}\right|=$ $\left|\beta_{j_{0}}\right|$. Consider $\alpha=\left(\alpha_{1}, \ldots, \alpha_{n}\right)$ with $\alpha_{j_{0}}=\frac{\overline{\beta_{j_{0}}}}{\left|\beta_{j_{0}}\right|}$ and $\alpha_{j}=0$ for $j \neq j_{0}$. For this choice we get

$$
\sum_{j=1}^{n}\left|\alpha_{j}\right|=\frac{\left|\overline{\beta_{j_{0}}}\right|}{\left|\beta_{j_{0}}\right|}=1 \text { and }\left|\sum_{j=1}^{n} \alpha_{j} \beta_{j}\right|=\left|\frac{\overline{\beta_{j_{0}}}}{\left|\beta_{j_{0}}\right|} \beta_{j_{0}}\right|=\left|\beta_{j_{0}}\right|=\|\beta\|_{\infty},
$$

therefore by (2.11) we obtain the representation

$$
\sup _{\|\alpha\|_{1} \leq 1}\left|\sum_{j=1}^{n} \alpha_{j} \beta_{j}\right|=\|\beta\|_{\infty}
$$

for any $\beta=\left(\beta_{1}, \ldots, \beta_{n}\right) \in \mathbb{K}^{n}$.

By taking the supremum in the equality (2.8) and by using the equality (2.12), we have

$$
\begin{aligned}
\sup _{\|\alpha\|_{1} \leq 1}\left\|\sum_{j=1}^{n} \alpha_{j} x_{j}\right\| & =\sup _{\|\alpha\|_{1} \leq 1}\left(\sup _{\|y\| \leq 1}\left|\sum_{j=1}^{n} \alpha_{j}\left\langle x_{j}, y\right\rangle\right|\right) \\
& =\sup _{\|f\| \leq 1}\left(\sup _{\|\alpha\|_{1} \leq 1}\left|\sum_{j=1}^{n} \alpha_{j}\left\langle x_{j}, y\right\rangle\right|\right)=\sup _{\|f\| \leq 1}\left(\max _{j \in\{1, \ldots, n\}}\left|\left\langle x_{j}, y\right\rangle\right|\right) \\
& =\max _{j \in\{1, \ldots, n\}}\left(\sup _{\|f\| \leq 1}\left|\left\langle x_{j}, y\right\rangle\right|\right)=\max _{j \in\{1, \ldots, n\}}\left\{\left\|x_{j}\right\|\right\},
\end{aligned}
$$

which proves (2.3). For the last equality we used the property $(2.7)$.

Corollary 1. With the assumptions of Theorem 3 we have for $q \geq 1$ that

$$
\frac{1}{n^{1 / q}}\|\mathbf{x}\|_{n, q} \leq\|\mathbf{x}\|_{h, n, q} \leq\|\mathbf{x}\|_{n, q}
$$

for any any $\mathbf{x} \in H^{n}$.

In particular, we have

$$
\frac{1}{\sqrt{n}}\|\mathbf{x}\|_{2} \leq\|\mathbf{x}\|_{h, e} \leq\|\mathbf{x}\|_{2}
$$

for any $\mathbf{x} \in H^{n}$.

Proof. Let $\mathbf{x} \in H^{n}$ with $\mathbf{x}=\left(x_{1}, \ldots, x_{n}\right)$ and $y \in H$ with $\|y\| \leq 1$, then for $q \geq 1$

$$
\left(\sum_{j=1}^{n}\left|\left\langle x_{j}, y\right\rangle\right|^{q}\right)^{1 / q} \leq\left(\sum_{j=1}^{n}\left(\|y\|\left\|x_{i}\right\|\right)^{q}\right)^{1 / q}=\|y\|\left(\sum_{j=1}^{n}\left\|x_{i}\right\|^{q}\right)^{1 / q}=\|y\|\|\mathbf{x}\|_{n, q}
$$

and by taking the supremum over $\|y\| \leq 1$, we get the second inequality in (2.13).

By the properties of complex numbers, we have

$$
\max _{j \in\{1, \ldots, n\}}\left\{\left|\left\langle x_{j}, y\right\rangle\right|\right\} \leq\left(\sum_{j=1}^{n}\left|\left\langle x_{j}, y\right\rangle\right|^{q}\right)^{1 / q}
$$


and by taking the supremum over $\|y\| \leq 1$, we get

$$
\sup _{\|y\| \leq 1}\left(\max _{j \in\{1, \ldots, n\}}\left\{\left|\left\langle x_{j}, y\right\rangle\right|\right\}\right) \leq \sup _{\|y\| \leq 1}\left(\sum_{j=1}^{n}\left|\left\langle x_{j}, y\right\rangle\right|^{q}\right)^{1 / q}
$$

and since

$$
\begin{aligned}
\sup _{\|y\| \leq 1}\left(\max _{j \in\{1, \ldots, n\}}\left\{\left|\left\langle x_{j}, y\right\rangle\right|\right\}\right) & =\max _{j \in\{1, \ldots, n\}}\left\{\sup _{\|y\| \leq 1}\left|\left\langle x_{j}, y\right\rangle\right|\right\} \\
& =\max _{j \in\{1, \ldots, n\}}\left\{\left\|x_{j}\right\|\right\}=\|\mathbf{x}\|_{n, \infty}
\end{aligned}
$$

then by (2.15) we get

$$
\|\mathbf{x}\|_{n, \infty} \leq\|\mathbf{x}\|_{h, n, q} \text { for any } \mathbf{x} \in H^{n} .
$$

Since

$$
\left(\sum_{j=1}^{n}\left\|x_{j}\right\|^{q}\right)^{1 / q} \leq\left(n\|\mathbf{x}\|_{n, \infty}^{q}\right)^{1 / q}=n^{1 / q}\|\mathbf{x}\|_{n, \infty}
$$

then also

$$
\frac{1}{n^{1 / q}}\|\mathbf{x}\|_{n, q} \leq\|\mathbf{x}\|_{n, \infty} \text { for any } \mathbf{x} \in H^{n}
$$

By utilising the inequalities (2.16) and (2.17) we obtain the first inequality in (2.13).

Remark 1. In the case of inner product spaces the inequality (2.14) has been obtained in a different and more difficult way in [9] by employing the rotationinvariant normalised positive Borel measure on the unit sphere.

Corollary 2. With the assumptions of Theorem 3 we have for $r \geq q \geq 1$ that

$$
\|\mathbf{x}\|_{h, n, r} \leq\|\mathbf{x}\|_{h, n, q} \leq n^{\frac{r-q}{r q}}\|\mathbf{x}\|_{h, n, r}
$$

for any any $\mathbf{x} \in H^{n}$.

In particular, for $q \geq 2$ we have

$$
\|\mathbf{x}\|_{h, n, q} \leq\|\mathbf{x}\|_{h, e} \leq n^{\frac{q-2}{2 q}}\|\mathbf{x}\|_{h, n, q}
$$

and for $1 \leq q \leq 2$ we have

$$
\|\mathbf{x}\|_{h, e} \leq\|\mathbf{x}\|_{h, n, q} \leq n^{\frac{2-q}{2 q}}\|\mathbf{x}\|_{h, e}
$$

for any $\mathbf{x} \in H^{n}$.

Proof. We use the following elementary inequalities for the nonnegative numbers $a_{j}, j=1, \ldots, n$ and $r \geq q>0$ (see for instance [16])

$$
\left(\sum_{j=1}^{n} a_{j}^{r}\right)^{1 / r} \leq\left(\sum_{j=1}^{n} a_{j}^{q}\right)^{1 / q} \leq n^{\frac{r-q}{r q}}\left(\sum_{j=1}^{n} a_{j}^{r}\right)^{1 / r}
$$

Let $\mathbf{x} \in H^{n}$ with $\mathbf{x}=\left(x_{1}, \ldots, x_{n}\right)$ and $y \in H$ with $\|y\| \leq 1$, then for $r \geq q \geq 1$ we have

$$
\left(\sum_{j=1}^{n}\left|\left\langle x_{j}, y\right\rangle\right|^{r}\right)^{1 / r} \leq\left(\sum_{j=1}^{n}\left|\left\langle x_{j}, y\right\rangle\right|^{q}\right)^{1 / q} \leq n^{\frac{r-q}{r q}}\left(\sum_{j=1}^{n}\left|\left\langle x_{j}, y\right\rangle\right|^{r}\right)^{1 / r} .
$$


By taking the supremum over $y \in H$ with $\|y\| \leq 1$ and using Theorem 3, we get (2.18).

Remark 2. If we take $q=1$ in (2.18), then we get

$$
\|\mathbf{x}\|_{h, n, r} \leq\|\mathbf{x}\|_{h, n, 1} \leq n^{\frac{r-1}{r}}\|\mathbf{x}\|_{h, n, r}
$$

for any any $\mathbf{x} \in H^{n}$.

In particular, for $r=2$ we get

$$
\|\mathbf{x}\|_{h, e} \leq\|\mathbf{x}\|_{h, n, 1} \leq \sqrt{n}\|\mathbf{x}\|_{h, e}
$$

for any any $\mathbf{x} \in H^{n}$.

\section{Some Reverse Inequalities}

Recall the following additive reverse of Cauchy-Buniakowski-Schwarz inequality [7] (see also [8, Theorem 5. 14])

Lemma 1. Let $a, A \in \mathbb{R}$ and $\mathbf{z}=\left(z_{1}, \ldots, z_{n}\right), \mathbf{y}=\left(y_{1}, \ldots, y_{n}\right)$ be two sequences of real numbers with the property that:

$$
a y_{j} \leq z_{j} \leq A y_{j} \text { for each } j \in\{1, \ldots, n\} .
$$

Then for any $\mathbf{w}=\left(w_{1}, \ldots, w_{n}\right)$ a sequence of positive real numbers, one has the inequality

$$
0 \leq \sum_{j=1}^{n} w_{j} z_{j}^{2} \sum_{j=1}^{n} w_{j} y_{j}^{2}-\left(\sum_{j=1}^{n} w_{j} z_{j} y_{j}\right)^{2} \leq \frac{1}{4}(A-a)^{2}\left(\sum_{j=1}^{n} w_{j} y_{j}^{2}\right)^{2} .
$$

The constant $\frac{1}{4}$ is sharp in (3.2).

O. Shisha and B. Mond obtained in 1967 (see [17]) the following counterparts of $(C B S)$ - inequality (see also [8, Theorem $5.20 \& 5.21]$ )

Lemma 2. Assume that $\mathbf{a}=\left(a_{1}, \ldots, a_{n}\right)$ and $\mathbf{b}=\left(b_{1}, \ldots, b_{n}\right)$ are such that there exists $a, A, b, B$ with the property that:

$$
0 \leq a \leq a_{j} \leq A \text { and } 0<b \leq b_{j} \leq B \text { for any } j \in\{1, \ldots, n\},
$$

then we have the inequality

$$
\sum_{j=1}^{n} a_{j}^{2} \sum_{j=1}^{n} b_{j}^{2}-\left(\sum_{j=1}^{n} a_{j} b_{j}\right)^{2} \leq\left(\sqrt{\frac{A}{b}}-\sqrt{\frac{a}{B}}\right)^{2} \sum_{j=1}^{n} a_{j} b_{j} \sum_{j=1}^{n} b_{j}^{2},
$$

and

Lemma 3. Assume that $\mathbf{a}, \mathbf{b}$ are nonnegative sequences and there exists $\gamma, \Gamma$ with the property that

$$
0 \leq \gamma \leq \frac{a_{j}}{b_{j}} \leq \Gamma<\infty \text { for any } j \in\{1, \ldots, n\} .
$$

Then we have the inequality

$$
0 \leq\left(\sum_{j=1}^{n} a_{j}^{2} \sum_{j=1}^{n} b_{j}^{2}\right)^{\frac{1}{2}}-\sum_{j=1}^{n} a_{j} b_{j} \leq \frac{(\Gamma-\gamma)^{2}}{4(\gamma+\Gamma)} \sum_{j=1}^{n} b_{j}^{2} .
$$

We have the following result: 
Theorem 4. Let $(H,\|\cdot\|)$ be an inner product space over the real or complex number field $\mathbb{K}$ and $\mathbf{x} \in H^{n}$ with $\mathbf{x}=\left(x_{1}, \ldots, x_{n}\right)$. Then we have

$$
\begin{gathered}
0 \leq\|\mathbf{x}\|_{h, e}^{2}-\frac{1}{n}\|\mathbf{x}\|_{h, n, 1}^{2} \leq \frac{1}{4} n\|\mathbf{x}\|_{n, \infty}^{2}, \\
0 \leq\|\mathbf{x}\|_{h, e}^{2}-\frac{1}{n}\|\mathbf{x}\|_{h, n, 1}^{2} \leq\|\mathbf{x}\|_{h, n, 1}\|\mathbf{x}\|_{n, \infty}
\end{gathered}
$$

and

$$
0 \leq\|\mathbf{x}\|_{h, e}-\frac{1}{\sqrt{n}}\|\mathbf{x}\|_{h, n, 1} \leq \frac{1}{4} \sqrt{n}\|\mathbf{x}\|_{n, \infty} .
$$

Proof. Let $\mathbf{x} \in H^{n}$ with $\mathbf{x}=\left(x_{1}, \ldots, x_{n}\right)$ and put $R=\max _{j \in\{1, \ldots, n\}}\left\{\left\|x_{j}\right\|\right\}=$ $\|\mathbf{x}\|_{n, \infty}$. If $y \in H$ with $\|y\| \leq 1$ then $\left|\left\langle x_{j}, y\right\rangle\right| \leq\|y\|\left\|x_{j}\right\| \leq R$ for any $j \in\{1, \ldots, n\}$.

If we write the inequality (3.2) for $z_{j}=\left|\left\langle x_{j}, y\right\rangle\right|, w_{j}=y_{j}=1, A=R$ and $a=0$, we get

$$
0 \leq n \sum_{j=1}^{n}\left|\left\langle x_{j}, y\right\rangle\right|^{2}-\left(\sum_{j=1}^{n}\left|\left\langle x_{j}, y\right\rangle\right|\right)^{2} \leq \frac{1}{4} n^{2} R^{2}
$$

for any $y \in H$ with $\|y\| \leq 1$.

This implies that

$$
\sum_{j=1}^{n}\left|\left\langle x_{j}, y\right\rangle\right|^{2} \leq \frac{1}{n}\left(\sum_{j=1}^{n}\left|\left\langle x_{j}, y\right\rangle\right|\right)^{2}+\frac{1}{4} n R^{2}
$$

for any $y \in H$ with $\|y\| \leq 1$.

By taking the supremum in (3.10) over $y \in H$ with $\|y\| \leq 1$ we get (3.7).

If we write the inequality (3.4) for $a_{j}=\left|\left\langle x_{j}, y\right\rangle\right|, b_{j}=1, b=B=1, a=0$ and $A=R$, then we get

$$
0 \leq n \sum_{j=1}^{n}\left|\left\langle x_{j}, y\right\rangle\right|^{2}-\left(\sum_{j=1}^{n}\left|\left\langle x_{j}, y\right\rangle\right|\right)^{2} \leq n R \sum_{j=1}^{n}\left|\left\langle x_{j}, y\right\rangle\right|,
$$

for any $y \in H$ with $\|y\| \leq 1$.

This implies that

$$
\sum_{j=1}^{n}\left|\left\langle x_{j}, y\right\rangle\right|^{2} \leq \frac{1}{n}\left(\sum_{j=1}^{n}\left|\left\langle x_{j}, y\right\rangle\right|\right)^{2}+R \sum_{j=1}^{n}\left|\left\langle x_{j}, y\right\rangle\right|,
$$

for any $y \in H$ with $\|y\| \leq 1$.

By taking the supremum in (3.11) over $y \in H$ with $\|y\| \leq 1$ we get (3.8).

Finally, if we write the inequality (3.6) for $a_{j}=\left|\left\langle x_{j}, y\right\rangle\right|, b_{j}=1, b=B=1$, $\gamma=0$ and $\Gamma=R$, then we have

$$
0 \leq\left(n \sum_{j=1}^{n}\left|\left\langle x_{j}, y\right\rangle\right|^{2}\right)^{\frac{1}{2}}-\sum_{j=1}^{n}\left|\left\langle x_{j}, y\right\rangle\right| \leq \frac{1}{4} n R,
$$

for any $y \in H$ with $\|y\| \leq 1$. 
This implies that

$$
\left(\sum_{j=1}^{n}\left|\left\langle x_{j}, y\right\rangle\right|^{2}\right)^{\frac{1}{2}} \leq \frac{1}{\sqrt{n}} \sum_{j=1}^{n}\left|\left\langle x_{j}, y\right\rangle\right|+\frac{1}{4} \sqrt{n} R,
$$

for any $y \in H$ with $\|y\| \leq 1$.

By taking the supremum in (3.12) over $y \in H$ with $\|y\| \leq 1$ we get (3.9).

Further, we recall the Čebyšev's inequality for synchronous $n$-tuples of vectors $\mathbf{a}=\left(a_{1}, \ldots, a_{n}\right)$ and $\mathbf{b}=\left(b_{1}, \ldots, b_{n}\right)$, namely if $\left(a_{j}-a_{k}\right)\left(b_{j}-b_{k}\right) \geq 0$ for any $j$, $k \in\{1, \ldots, n\}$, then

$$
\frac{1}{n} \sum_{j=1}^{n} a_{j} b_{j} \geq \frac{1}{n} \sum_{j=1}^{n} a_{j} \frac{1}{n} \sum_{j=1}^{n} b_{j}
$$

In 1950, Biernacki et al. [2] obtained the following discrete version of Grüss' inequality:

Lemma 4. Assume that $\mathbf{a}=\left(a_{1}, \ldots, a_{n}\right)$ and $\mathbf{b}=\left(b_{1}, \ldots, b_{n}\right)$ are such that there exists real numbers $a, A, b, B$ with the property that:

$$
a \leq a_{j} \leq A \text { and } b \leq b_{j} \leq B \text { for any } j \in\{1, \ldots, n\} .
$$

Then

$$
\begin{aligned}
& \left|\frac{1}{n} \sum_{j=1}^{n} a_{j} b_{j}-\frac{1}{n} \sum_{j=1}^{n} a_{j} \frac{1}{n} \sum_{j=1}^{n} b_{j}\right| \\
& \leq \frac{1}{n}\left\lceil\frac{n}{2}\right\rceil\left(1-\frac{1}{n}\left\lceil\frac{n}{2}\right\rceil\right)(A-a)(B-b) \\
& =\frac{1}{n^{2}}\left\lceil\frac{n^{2}}{4}\right\rceil(A-a)(B-a) \leq \frac{1}{4}(A-a)(B-b),
\end{aligned}
$$

where $\lceil x\rceil$ gives the largest integer less than or equal to $x$.

The following result also holds:

Theorem 5. Let $(H,\|\cdot\|)$ be an inner product space over the real or complex number field $\mathbb{K}$ and $\mathbf{x} \in H^{n}$ with $\mathbf{x}=\left(x_{1}, \ldots, x_{n}\right)$. Then for $q, r \geq 1$ we have

$$
\begin{aligned}
\|\mathbf{x}\|_{h, n, q+r}^{q+r} & \leq \frac{1}{n}\|\mathbf{x}\|_{h, n, q}^{q}\|\mathbf{x}\|_{h, n, r}^{r}+\frac{1}{n}\left\lceil\frac{n^{2}}{4}\right\rceil\|\mathbf{x}\|_{n, \infty}^{q+r} \\
& \leq \frac{1}{n}\|\mathbf{x}\|_{h, n, q}^{q}\|\mathbf{x}\|_{h, n, r}^{r}+\frac{1}{4} n\|\mathbf{x}\|_{n, \infty}^{q+r} .
\end{aligned}
$$

Proof. Let $\mathbf{x} \in H^{n}$ with $\mathbf{x}=\left(x_{1}, \ldots, x_{n}\right)$ and put $R=\max _{j \in\{1, \ldots, n\}}\left\{\left\|x_{j}\right\|\right\}=$ $\|\mathbf{x}\|_{n, \infty}$. If $f \in H$ with $\|y\| \leq 1$ then $\left|\left\langle x_{j}, y\right\rangle\right| \leq\|y\|\left\|x_{j}\right\| \leq R$ for any $j \in\{1, \ldots, n\}$.

If we take into the inequality (3.15) $a_{j}=\left|\left\langle x_{j}, y\right\rangle\right|^{q}, b_{j}=\left|\left\langle x_{j}, y\right\rangle\right|^{r}, a=0, A=R^{q}$, $b=0$ and $B=R^{r}$, then we get

$$
\left.\left|\frac{1}{n} \sum_{j=1}^{n}\right|\left\langle x_{j}, y\right\rangle\right|^{q+r}-\frac{1}{n} \sum_{j=1}^{n}\left|\left\langle x_{j}, y\right\rangle\right|^{q} \frac{1}{n} \sum_{j=1}^{n}\left|\left\langle x_{j}, y\right\rangle\right|^{r} \mid \leq \frac{1}{n^{2}}\left\lceil\frac{n^{2}}{4}\right\rceil R^{q+r} .
$$


On the other hand, since the sequences $\left\{a_{j}\right\}_{j=1, \ldots, n},\left\{b_{j}\right\}_{j=1, \ldots, n}$ are synchronous, then by (3.13) we have

$$
0 \leq \frac{1}{n} \sum_{j=1}^{n}\left|\left\langle x_{j}, y\right\rangle\right|^{q+r}-\frac{1}{n} \sum_{j=1}^{n}\left|\left\langle x_{j}, y\right\rangle\right|^{q} \frac{1}{n} \sum_{j=1}^{n}\left|\left\langle x_{j}, y\right\rangle\right|^{r} .
$$

Using (3.17) we then get

$$
\sum_{j=1}^{n}\left|\left\langle x_{j}, y\right\rangle\right|^{q+r} \leq \frac{1}{n} \sum_{j=1}^{n}\left|\left\langle x_{j}, y\right\rangle\right|^{q} \sum_{j=1}^{n}\left|\left\langle x_{j}, y\right\rangle\right|^{r}+\frac{1}{n}\left\lceil\frac{n^{2}}{4}\right\rceil R^{q+r}
$$

for any $y \in H$ with $\|y\| \leq 1$.

By taking the supremum in (3.18), we get

$$
\begin{aligned}
& \sup _{\|y\| \leq 1}\left\{\sum_{j=1}^{n}\left|\left\langle x_{j}, y\right\rangle\right|^{q+r}\right\} \\
& \leq \frac{1}{n} \sup _{\|y\| \leq 1}\left\{\sum_{j=1}^{n}\left|\left\langle x_{j}, y\right\rangle\right|^{q} \sum_{j=1}^{n}\left|\left\langle x_{j}, y\right\rangle\right|^{r}\right\}+\frac{1}{n}\left\lceil\frac{n^{2}}{4}\right\rceil R^{q+r} \\
& \leq \frac{1}{n} \sup _{\|y\| \leq 1}\left\{\sum_{j=1}^{n}\left|\left\langle x_{j}, y\right\rangle\right|^{q}\right\} \sup _{\|y\| \leq 1}\left\{\sum_{j=1}^{n}\left|\left\langle x_{j}, y\right\rangle\right|^{r}\right\}+\frac{1}{n}\left\lceil\frac{n^{2}}{4}\right\rceil R^{q+r},
\end{aligned}
$$

which proves the first inequality in (3.16).

The second part of (3.16) is obvious.

Corollary 3. With the assumptions of Theorem 5 and if $r \geq 1$, then we have

$$
\|\mathbf{x}\|_{h, n, 2 r}^{2 r} \leq \frac{1}{n}\|\mathbf{x}\|_{h, n, r}^{2 r}+\frac{1}{n}\left\lceil\frac{n^{2}}{4}\right\rceil\|\mathbf{x}\|_{n, \infty}^{2 r} \leq \frac{1}{n}\|\mathbf{x}\|_{h, n, r}^{2 r}+\frac{1}{4} n\|\mathbf{x}\|_{n, \infty}^{2 r} .
$$

In particular, for $r=1$ we get

$$
\|\mathbf{x}\|_{h, e}^{2} \leq \frac{1}{n}\|\mathbf{x}\|_{h, n, 1}^{2}+\frac{1}{n}\left\lceil\frac{n^{2}}{4}\right\rceil\|\mathbf{x}\|_{n, \infty}^{2} \leq \frac{1}{n}\|\mathbf{x}\|_{h, n, 1}^{2}+\frac{1}{4} n\|\mathbf{x}\|_{n, \infty}^{2} .
$$

The first inequality in (3.20) is better than the second inequality in (3.7).

For an $n$-tuple of complex numbers $\mathbf{a}=\left(a_{1}, \ldots, a_{n}\right)$ with $n \geq 2$ consider the $(n-1)$-tuple built by the aid of forward differences $\Delta \mathbf{a}=\left(\Delta a_{1}, \ldots, \Delta a_{n-1}\right)$ where $\Delta a_{k}:=a_{k+1}-a_{k}$ where $k \in\{1, \ldots, n-1\}$. Similarly, if $\mathbf{x}=\left(x_{1}, \ldots, x_{n}\right) \in H^{n}$ is an $n$-tuple of vectors we also can consider in a similar way the $(n-1)$-tuple $\Delta \mathbf{x}=\left(\Delta x_{1}, \ldots, \Delta x_{n-1}\right)$.

We obtained the following Grüss' type inequalities in terms of forward differences: 
Lemma 5. Assume that $\mathbf{a}=\left(a_{1}, \ldots, a_{n}\right)$ and $\mathbf{b}=\left(b_{1}, \ldots, b_{n}\right)$ are $n$-tuples of complex numbers. Then

$$
\begin{aligned}
& \left|\frac{1}{n} \sum_{j=1}^{n} a_{j} b_{j}-\frac{1}{n} \sum_{j=1}^{n} a_{j} \frac{1}{n} \sum_{j=1}^{n} b_{j}\right| \\
& \leq\left\{\begin{array}{l}
\frac{1}{12}\left(n^{2}-1\right)\|\Delta \mathbf{a}\|_{n-1, \infty}\|\Delta \mathbf{b}\|_{n-1, \infty}, \quad[12], \\
\frac{1}{6} \frac{n^{2}-1}{n}\|\Delta \mathbf{a}\|_{n-1, \alpha}\|\Delta \mathbf{b}\|_{n-1, \beta} \text { where } \alpha, \beta>1, \frac{1}{\alpha}+\frac{1}{\beta}=1, \quad[5], \\
\frac{1}{2}\left(1-\frac{1}{n}\right)\|\Delta \mathbf{a}\|_{n-1,1}\|\Delta \mathbf{b}\|_{n-1,1}, \quad[6] .
\end{array}\right.
\end{aligned}
$$

The constants $\frac{1}{12}, \frac{1}{6}$ and $\frac{1}{2}$ are best possible in (3.21).

The following result also holds:

Theorem 6. Let $(H,\langle\cdot, \cdot\rangle)$ be an inner product space over the real or complex number field $\mathbb{K}$ and $\mathbf{x} \in H^{n}$ with $\mathbf{x}=\left(x_{1}, \ldots, x_{n}\right)$. Then for $q, r \geq 1$ we have

$$
\begin{aligned}
\|\mathbf{x}\|_{h, n, q+r}^{q+r} \leq & \frac{1}{n}\|\mathbf{x}\|_{h, n, q}^{q}\|\mathbf{x}\|_{h, n, r}^{r} \\
+ & \left\{\begin{array}{l}
\frac{1}{12} q r\left(n^{2}-1\right) n\|\mathbf{x}\|_{n, \infty}^{q+r-2}\|\Delta \mathbf{x}\|_{n-1, \infty}^{2}, \\
\frac{1}{6}\left(n^{2}-1\right) q r\|\mathbf{x}\|_{n, \infty}^{q+r-2}\|\Delta \mathbf{x}\|_{h, n-1, \alpha}\|\Delta \mathbf{x}\|_{h, n-1, \beta} \\
\text { where } \alpha, \beta>1, \frac{1}{\alpha}+\frac{1}{\beta}=1, \\
\frac{1}{2}(n-1) q r\|\mathbf{x}\|_{n, \infty}^{q+r-2}\|\Delta \mathbf{x}\|_{h, n-1,1}^{2} .
\end{array}\right.
\end{aligned}
$$

Proof. Let $\mathbf{x} \in H^{n}$ with $\mathbf{x}=\left(x_{1}, \ldots, x_{n}\right)$ and $y \in H$ with $\|y\| \leq 1$. If we take into the inequality (3.21) $a_{j}=\left|\left\langle x_{j}, y\right\rangle\right|^{q}, b_{j}=\left|\left\langle x_{j}, y\right\rangle\right|^{r}$, then we get

$$
\begin{aligned}
& \left.\left|\frac{1}{n} \sum_{j=1}^{n}\right|\left\langle x_{j}, y\right\rangle\right|^{q+r}-\frac{1}{n} \sum_{j=1}^{n}\left|\left\langle x_{j}, y\right\rangle\right|^{q} \frac{1}{n} \sum_{j=1}^{n}\left|\left\langle x_{j}, y\right\rangle\right|^{r} \mid \\
& \leq\left\{\begin{array}{l}
\left.\frac{1}{12}\left(n^{2}-1\right) \max _{j=1, \ldots, n-1}|\Delta|\left\langle x_{j}, y\right\rangle\right|^{q}\left|\max _{j=1, \ldots, n-1}\right| \Delta\left|\left\langle x_{j}, y\right\rangle\right|^{r} \mid, \\
\frac{1}{6} \frac{n^{2}-1}{n}\left(\left.\left.\sum_{j=1}^{n-1}|\Delta|\left\langle x_{j}, y\right\rangle\right|^{q}\right|^{\alpha}\right)^{1 / \alpha}\left(\left.\left.\sum_{j=1}^{n-1}|\Delta|\left\langle x_{j}, y\right\rangle\right|^{r}\right|^{\beta}\right)^{1 / \beta} \\
\text { where } \alpha, \beta>1, \frac{1}{\alpha}+\frac{1}{\beta}=1, \\
\left.\frac{1}{2}\left(1-\frac{1}{n}\right) \sum_{j=1}^{n-1}|\Delta|\left\langle x_{j}, y\right\rangle\right|^{q}\left|\sum_{j=1}^{n-1}\right| \Delta\left|\left\langle x_{j}, y\right\rangle\right|^{r} \mid .
\end{array}\right.
\end{aligned}
$$

We use the following elementary inequality for powers $p \geq 1$

$$
\left|a^{p}-b^{p}\right| \leq p R^{p-1}|a-b|
$$

where $a, b \in[0, R]$.

Put $R=\max _{j \in\{1, \ldots, n\}}\left\{\left\|x_{j}\right\|\right\}=\|\mathbf{x}\|_{n, \infty}$. Then for any $y \in H$ with $\|y\| \leq 1$ we have $\left|\left\langle x_{j}, y\right\rangle\right| \leq\|y\|\left\|x_{j}\right\| \leq R$ for any $j \in\{1, \ldots, n\}$.

Therefore

$$
\begin{aligned}
\left.|\Delta|\left\langle x_{j}, y\right\rangle\right|^{q} \mid & =\left.||\left\langle x_{j+1}, y\right\rangle\right|^{q}-\left|\left\langle x_{j}, y\right\rangle\right|^{q}\left|\leq q R^{q-1}\right|\left|\left\langle x_{j+1}, y\right\rangle\right|-\left|\left\langle x_{j}, y\right\rangle\right| \mid \\
& \leq q R^{q-1}\left|\left\langle x_{j+1}, y\right\rangle-\left\langle x_{j}, y\right\rangle\right|=q R^{q-1}\left|\left\langle\Delta x_{j}, y\right\rangle\right|
\end{aligned}
$$


for any $j=1, \ldots, n-1$, where $\Delta x_{j}=x_{j+1}-x_{j}$ is the forward difference.

On the other hand, since the sequences $\left\{a_{j}\right\}_{j=1, \ldots, n},\left\{b_{j}\right\}_{j=1, \ldots, n}$ are synchronous, then we have

$$
0 \leq \frac{1}{n} \sum_{j=1}^{n}\left|\left\langle x_{j}, y\right\rangle\right|^{q+r}-\frac{1}{n} \sum_{j=1}^{n}\left|\left\langle x_{j}, y\right\rangle\right|^{q} \frac{1}{n} \sum_{j=1}^{n}\left|\left\langle x_{j}, y\right\rangle\right|^{r}
$$

and by the first inequality in (3.23) we get

$$
\begin{aligned}
& \sum_{j=1}^{n}\left|\left\langle x_{j}, y\right\rangle\right|^{q+r} \\
& \leq \frac{1}{n} \sum_{j=1}^{n}\left|\left\langle x_{j}, y\right\rangle\right|^{q} \sum_{j=1}^{n}\left|\left\langle x_{j}, y\right\rangle\right|^{r} \\
& +\frac{1}{12}\left(n^{2}-1\right) n q R^{q-1} \max _{j=1, \ldots, n-1}\left|\left\langle\Delta x_{j}, y\right\rangle\right| r R^{r-1} \max _{j=1, \ldots, n-1}\left|\left\langle\Delta x_{j}, y\right\rangle\right| \\
& =\frac{1}{n} \sum_{j=1}^{n}\left|\left\langle\Delta x_{j}, y\right\rangle\right|^{q} \sum_{j=1}^{n}\left|\left\langle\Delta x_{j}, y\right\rangle\right|^{r} \\
& +\frac{1}{12}\left(n^{2}-1\right) n q r R^{q+r-2}\left(_{j=1, \ldots, n-1}^{\left.\max _{j}\left|\left\langle\Delta x_{j}, y\right\rangle\right|\right)^{2}}\right.
\end{aligned}
$$

for any $y \in H$ with $\|y\| \leq 1$.

Taking the supremum over $y \in H$ with $\|y\| \leq 1$ in (3.26) we get the first branch in the inequality (3.22).

We also have, by (3.24), that

$$
\begin{aligned}
\left(\left.\left.\sum_{j=1}^{n-1}|\Delta|\left\langle x_{j}, y\right\rangle\right|^{q}\right|^{\alpha}\right)^{1 / \alpha} & \leq\left[\left(q R^{q-1}\right)^{\alpha} \sum_{j=1}^{n-1}\left|\left\langle\Delta x_{j}, y\right\rangle\right|^{\alpha}\right]^{1 / \alpha} \\
& =q R^{q-1}\left(\sum_{j=1}^{n-1}\left|\left\langle\Delta x_{j}, y\right\rangle\right|^{\alpha}\right)^{1 / \alpha}
\end{aligned}
$$

and, similarly,

$$
\left(\left.\left.\sum_{j=1}^{n-1}|\Delta|\left\langle x_{j}, y\right\rangle\right|^{r}\right|^{\beta}\right)^{1 / \beta} \leq r R^{r-1}\left(\sum_{j=1}^{n-1}\left|\left\langle\Delta x_{j}, y\right\rangle\right|^{\beta}\right)^{1 / \beta}
$$

where $\alpha, \beta>1, \frac{1}{\alpha}+\frac{1}{\beta}=1$. 
By the second inequality in (3.23) and by (3.25) we have

$$
\begin{aligned}
& \sum_{j=1}^{n}\left|\left\langle x_{j}, y\right\rangle\right|^{q+r} \\
& \leq \frac{1}{n} \sum_{j=1}^{n}\left|\left\langle x_{j}, y\right\rangle\right|^{q} \sum_{j=1}^{n}\left|\left\langle x_{j}, y\right\rangle\right|^{r} \\
& +\frac{1}{6}\left(n^{2}-1\right)\left(\left.\left.\sum_{j=1}^{n-1}|\Delta|\left\langle x_{j}, y\right\rangle\right|^{q}\right|^{\alpha}\right)^{1 / \alpha}\left(\left.\left.\sum_{j=1}^{n-1}|\Delta|\left\langle x_{j}, y\right\rangle\right|^{r}\right|^{\beta}\right)^{1 / \beta} \\
& \leq \frac{1}{n} \sum_{j=1}^{n}\left|\left\langle x_{j}, y\right\rangle\right|^{q} \sum_{j=1}^{n}\left|\left\langle x_{j}, y\right\rangle\right|^{r} \\
& +\frac{1}{6}\left(n^{2}-1\right) q r R^{q+r-2}\left(\sum_{j=1}^{n-1}\left|\left\langle\Delta x_{j}, y\right\rangle\right|^{\alpha}\right)^{1 / \alpha}\left(\sum_{j=1}^{n-1}\left|\left\langle\Delta x_{j}, y\right\rangle\right|^{\beta}\right)^{1 / \beta}
\end{aligned}
$$

for any $y \in H$ with $\|y\| \leq 1$, where $\alpha, \beta>1, \frac{1}{\alpha}+\frac{1}{\beta}=1$.

Taking the supremum over $y \in H$ with $\|y\| \leq 1$ in (3.27) we get the second branch in the inequality (3.22).

We also have, by (3.24), that

$$
\left.\sum_{j=1}^{n-1}|\Delta|\left\langle x_{j}, y\right\rangle\right|^{q}\left|\leq q R^{q-1} \sum_{j=1}^{n-1}\right|\left\langle\Delta x_{j}, y\right\rangle \mid
$$

and

$$
\left.\sum_{j=1}^{n-1}|\Delta|\left\langle x_{j}, y\right\rangle\right|^{r}\left|\leq r R^{r-1} \sum_{j=1}^{n-1}\right|\left\langle\Delta x_{j}, y\right\rangle \mid .
$$

By the third inequality in (3.23) and by (3.25) we have

$$
\begin{aligned}
\sum_{j=1}^{n}\left|\left\langle x_{j}, y\right\rangle\right|^{q+r} & \leq \frac{1}{n} \sum_{j=1}^{n}\left|\left\langle x_{j}, y\right\rangle\right|^{q} \sum_{j=1}^{n}\left|\left\langle x_{j}, y\right\rangle\right|^{r} \\
& +\left.\frac{1}{2}(n-1) \sum_{j=1}^{n-1}|\Delta|\left\langle x_{j}, y\right\rangle\right|^{q}\left|\sum_{j=1}^{n-1}\right| \Delta\left|\left\langle x_{j}, y\right\rangle\right|^{r} \mid \\
& \leq \frac{1}{n} \sum_{j=1}^{n}\left|\left\langle x_{j}, y\right\rangle\right|^{q} \sum_{j=1}^{n}\left|\left\langle x_{j}, y\right\rangle\right|^{r} \\
& +\frac{1}{2}(n-1) q r R^{q+r-2} \sum_{j=1}^{n-1}\left|\left\langle\Delta x_{j}, y\right\rangle\right| \sum_{j=1}^{n-1}\left|\left\langle\Delta x_{j}, y\right\rangle\right|
\end{aligned}
$$

for any $y \in H$ with $\|y\| \leq 1$.

Taking the supremum over $y \in H$ with $\|y\| \leq 1$ in (3.28) we get the third branch in the inequality (3.22). 
Corollary 4. With the assumptions of Theorem 6 and if $r \geq 1$, then we have

$$
\begin{aligned}
\|\mathbf{x}\|_{h, n, 2 r}^{2 r} \leq & \frac{1}{n}\|\mathbf{x}\|_{h, n, r}^{2 r} \\
+ & \left\{\begin{array}{l}
\frac{1}{12} r^{2}\left(n^{2}-1\right) n\|\mathbf{x}\|_{n, \infty}^{2 r-2}\|\Delta \mathbf{x}\|_{n-1, \infty}^{2} \\
\frac{1}{6} r^{2}\left(n^{2}-1\right)\|\mathbf{x}\|_{n, \infty}^{2 r-2}\|\Delta \mathbf{x}\|_{h, n-1, \alpha}\|\Delta \mathbf{x}\|_{h, n-1, \beta} \\
\text { where } \alpha, \beta>1, \frac{1}{\alpha}+\frac{1}{\beta}=1, \\
\frac{1}{2} r^{2}(n-1)\|\mathbf{x}\|_{n, \infty}^{2 r-2}\|\Delta \mathbf{x}\|_{h, n-1,1}^{2} .
\end{array}\right.
\end{aligned}
$$

In particular, for $r=1$ we get

$$
\|\mathbf{x}\|_{h, e}^{2} \leq \frac{1}{n}\|\mathbf{x}\|_{h, n, 1}^{2}+\left\{\begin{array}{l}
\frac{1}{12}\left(n^{2}-1\right) n\|\Delta \mathbf{x}\|_{n-1, \infty}^{2} \\
\frac{1}{6}\left(n^{2}-1\right)\|\Delta \mathbf{x}\|_{h, n-1, \alpha}\|\Delta \mathbf{x}\|_{h, n-1, \beta} \\
\text { where } \alpha, \beta>1, \frac{1}{\alpha}+\frac{1}{\beta}=1, \\
\frac{1}{2}(n-1)\|\Delta \mathbf{x}\|_{h, n-1,1}^{2} .
\end{array} .\right.
$$

\section{Further Inequalities}

In 1992, J. Pečarić [15] proved the following general inequality in inner product spaces:

Lemma 6. Let $y, x_{1}, \ldots, x_{n} \in H$ and $c_{1}, \ldots, c_{n} \in \mathbb{K}$. Then

$$
\begin{aligned}
\left|\sum_{i=1}^{n} c_{i}\left\langle x_{i}, y\right\rangle\right|^{2} & \leq\|y\|^{2} \sum_{i=1}^{n}\left|c_{i}\right|^{2}\left(\sum_{j=1}^{n}\left|\left\langle x_{i}, x_{j}\right\rangle\right|\right) \\
& \leq\|y\|^{2} \sum_{i=1}^{n}\left|c_{i}\right|^{2} \max _{1 \leq i \leq n}\left\{\sum_{j=1}^{n}\left|\left\langle x_{i}, x_{j}\right\rangle\right|\right\} .
\end{aligned}
$$

He showed that the Bombieri inequality [4] may be obtained from (4.1) for the choice $c_{i}=\overline{\left\langle x_{i}, y\right\rangle}$ (using the second inequality), the Selberg inequality [14, p. 394] may be obtained from the first part of (4.1) for the choice

$$
c_{i}=\frac{\overline{\left\langle x_{i}, y\right\rangle}}{\sum_{j=1}^{n}\left|\left\langle x_{i}, x_{j}\right\rangle\right|}, \quad i \in\{1, \ldots, n\}
$$

while the Heilbronn inequality [13] may be obtained from the first part of (4.1) if one chooses $c_{i}=\frac{\overline{\left\langle x_{i}, y\right\rangle}}{\left|\left\langle x_{i}, y\right\rangle\right|}$, for any $i \in\{1, \ldots, n\}$.

Theorem 7. Let $(H,\langle\cdot, \cdot\rangle)$ be an inner product space over the real or complex number field $\mathbb{K}$ and $\mathbf{x} \in H^{n}$ with $\mathbf{x}=\left(x_{1}, \ldots, x_{n}\right)$. Then for $q \geq 1$ we have

$$
\|\mathbf{x}\|_{h, n, q} \leq\|\mathbf{x}\|_{h, n, 2(q-1)}^{1-1 / q}\left[\max _{1 \leq i \leq n}\left\{\sum_{j=1}^{n}\left|\left\langle x_{i}, x_{j}\right\rangle\right|\right\}\right]^{\frac{1}{2 q}}
$$


In particular, for $q=1$ we get

$$
\|\mathbf{x}\|_{h, n, 1} \leq\left[\max _{1 \leq i \leq n}\left\{\sum_{j=1}^{n}\left|\left\langle x_{i}, x_{j}\right\rangle\right|\right\}\right]^{\frac{1}{2}}
$$

while for $q=2$ we get

$$
\|\mathbf{x}\|_{h, e} \leq\left[\max _{1 \leq i \leq n}\left\{\sum_{j=1}^{n}\left|\left\langle x_{i}, x_{j}\right\rangle\right|\right\}\right]^{\frac{1}{2}} .
$$

Proof. If we take in (4.1) $c_{i}=\overline{\left\langle x_{i}, y\right\rangle}\left|\left\langle x_{i}, y\right\rangle\right|^{q-2}, i \in\{1, \ldots, n\}$, then we get

$$
\left(\sum_{i=1}^{n}\left|\left\langle x_{i}, y\right\rangle\right|^{q}\right)^{2} \leq\|y\|^{2} \sum_{i=1}^{n}\left|\left\langle x_{i}, y\right\rangle\right|^{2(q-1)} \max _{1 \leq i \leq n}\left\{\sum_{j=1}^{n}\left|\left\langle x_{i}, x_{j}\right\rangle\right|\right\}
$$

for any $\mathbf{x} \in H^{n}$ with $\mathbf{x}=\left(x_{1}, \ldots, x_{n}\right)$ and $y \in H$.

By taking the square root in (4.5) we get

$$
\begin{aligned}
& \sum_{i=1}^{n}\left|\left\langle x_{i}, y\right\rangle\right|^{q} \\
& \leq\|y\|\left(\sum_{i=1}^{n}\left|\left\langle x_{i}, y\right\rangle\right|^{2(q-1)}\right)^{1 / 2}\left[\max _{1 \leq i \leq n}\left\{\sum_{j=1}^{n}\left|\left\langle x_{i}, x_{j}\right\rangle\right|\right\}\right]^{1 / 2},
\end{aligned}
$$

for any $\mathbf{x} \in H^{n}$ with $\mathbf{x}=\left(x_{1}, \ldots, x_{n}\right)$ and $y \in H$.

If we take the supremum in (4.6) for $\|y\| \leq 1$, then we get

$$
\|\mathbf{x}\|_{h, n, q}^{q} \leq\|\mathbf{x}\|_{h, n, 2(q-1)}^{q-1}\left[\max _{1 \leq i \leq n}\left\{\sum_{j=1}^{n}\left|\left\langle x_{i}, x_{j}\right\rangle\right|\right\}\right]^{1 / 2}
$$

for any $\mathbf{x} \in H^{n}$ with $\mathbf{x}=\left(x_{1}, \ldots, x_{n}\right)$ and $y \in H$.

This proves (4.2).

In 1941, R. P. Boas [3] and in 1944, independently, R. Bellman [1] proved the following generalization of Bessel's inequality

Lemma 7. If $y, x_{1}, \ldots, x_{n}$ are elements of an inner product space $(H ;\langle\cdot, \cdot\rangle)$, then the following inequality:

$$
\sum_{i=1}^{n}\left|\left\langle x_{i}, y\right\rangle\right|^{2} \leq\|y\|^{2}\left[\max _{1 \leq i \leq n}\left\|x_{i}\right\|^{2}+\left(\sum_{1 \leq i \neq j \leq n}\left|\left\langle x_{i}, x_{j}\right\rangle\right|^{2}\right)^{\frac{1}{2}}\right],
$$

holds.

A generalization of the Boas-Bellman result was given in Mitrinović-Pečarić-Fink [14, p. 392] where they proved the following: 
Lemma 8. If $y, x_{1}, \ldots, x_{n}$ are as in Lemma 7 and $c_{1}, \ldots, c_{n} \in \mathbb{K}$, then one has the inequality:

$$
\left|\sum_{i=1}^{n} c_{i}\left\langle x_{i}, y\right\rangle\right|^{2} \leq\|y\|^{2} \sum_{i=1}^{n}\left|c_{i}\right|^{2}\left[\max _{1 \leq i \leq n}\left\|x_{i}\right\|^{2}+\left(\sum_{1 \leq i \neq j \leq n}\left|\left\langle x_{i}, x_{j}\right\rangle\right|^{2}\right)^{\frac{1}{2}}\right]
$$

They also noted that if in (4.8) one chooses $c_{i}=\overline{\left(x, y_{i}\right)}$, then this inequality becomes (4.7).

Using a similar argument to the one in Theorem 7 and Lemma 8 we have:

Theorem 8. Let $(H,\langle\cdot, \cdot\rangle)$ be an inner product space over the real or complex number field $\mathbb{K}$ and $\mathbf{x} \in H^{n}$ with $\mathbf{x}=\left(x_{1}, \ldots, x_{n}\right)$. Then for $q \geq 1$ we have

$$
\|\mathbf{x}\|_{h, n, q} \leq\|\mathbf{x}\|_{h, n, 2(q-1)}^{1-1 / q}\left[\max _{1 \leq i \leq n}\left\|x_{i}\right\|^{2}+\left(\sum_{1 \leq i \neq j \leq n}\left|\left\langle x_{i}, x_{j}\right\rangle\right|^{2}\right)^{\frac{1}{2}}\right]^{\frac{1}{2 q}} .
$$

In particular, for $q=1$ we get

$$
\|\mathbf{x}\|_{h, n, 1} \leq\left[\max _{1 \leq i \leq n}\left\|x_{i}\right\|^{2}+\left(\sum_{1 \leq i \neq j \leq n}\left|\left\langle x_{i}, x_{j}\right\rangle\right|^{2}\right)^{\frac{1}{2}}\right]^{\frac{1}{2}}
$$

while for $q=2$ we get

$$
\|\mathbf{x}\|_{h, e} \leq\left[\max _{1 \leq i \leq n}\left\|x_{i}\right\|^{2}+\left(\sum_{1 \leq i \neq j \leq n}\left|\left\langle x_{i}, x_{j}\right\rangle\right|^{2}\right)^{\frac{1}{2}}\right]^{\frac{1}{2}} .
$$

In [10] we obtained the following result that provides some companions to the Boas-Bellman inequality above:

Lemma 9. Let $y, x_{1}, \ldots, x_{n} \in H$ and $c_{1}, \ldots, c_{n} \in \mathbb{K}$. Then

$$
\begin{aligned}
& \left|\sum_{i=1}^{n} c_{i}\left\langle x_{i}, y\right\rangle\right|^{2} \\
& \leq\|y\|^{2} \times\left\{\begin{array}{l}
\max _{1 \leq i \leq n}\left\{\left|c_{i}\right|^{2}\right\}\left\{\sum_{i=1}^{n}\left\|x_{i}\right\|^{2}+\sum_{1 \leq i \neq j \leq n}\left|\left\langle x_{i}, x_{j}\right\rangle\right|\right\} \\
\left(\sum_{i=1}^{n}\left|c_{i}\right|^{2 p}\right)^{\frac{1}{p}}\left\{\left(\sum_{i=1}^{n}\left\|x_{i}\right\|^{2 r}\right)^{\frac{1}{r}}\right. \\
\left.+(n-1)^{\frac{1}{p}}\left(\sum_{1 \leq i \neq j \leq n}\left|\left\langle x_{i}, x_{j}\right\rangle\right|^{r}\right)^{\frac{1}{r}}\right\} \\
\text { where } p>1, \frac{1}{p}+\frac{1}{r}=1 \\
\sum_{i=1}^{n}\left|c_{i}\right|^{2}\left\{\max _{1 \leq i \leq n}\left\|x_{i}\right\|^{2}+(n-1) \max _{1 \leq i \neq j \leq n}\left|\left\langle x_{i}, x_{j}\right\rangle\right|\right\} .
\end{array}\right.
\end{aligned}
$$

We have: 
Theorem 9. Let $(H,\langle\cdot, \cdot\rangle)$ be an inner product space over the real or complex number field $\mathbb{K}$ and $\mathbf{x} \in H^{n}$ with $\mathbf{x}=\left(x_{1}, \ldots, x_{n}\right)$. Then for $q \geq 1$ we have

$$
\|\mathbf{x}\|_{h, n, q} \leq\left\{\begin{array}{l}
\max _{1 \leq i \leq n}\left\{\left\|x_{i}\right\|\right\}^{1-1 / q}\left\{\sum_{i=1}^{n}\left\|x_{i}\right\|^{2}+\sum_{1 \leq i \neq j \leq n}\left|\left\langle x_{i}, x_{j}\right\rangle\right|\right\}^{\frac{1}{2 q}} \\
\|\mathbf{x}\|_{h, n, 2 p(q-1)}^{1-1 / q}\left\{\left(\sum_{i=1}^{n}\left\|x_{i}\right\|^{2 r}\right)^{\frac{1}{r}}\right. \\
\left.+(n-1)^{\frac{1}{p}}\left(\sum_{1 \leq i \neq j \leq n}\left|\left\langle x_{i}, x_{j}\right\rangle\right|^{r}\right)^{\frac{1}{r}}\right\}^{\frac{1}{2 q}}, \\
\text { where p>1, } \frac{1}{p}+\frac{1}{r}=1 \\
\|\mathbf{x}\|_{h, n, 2(q-1)}^{1-1 / q}\left\{\max _{1 \leq i \leq n}\left\|x_{i}\right\|^{2}+(n-1) \max _{1 \leq i \neq j \leq n}\left|\left\langle x_{i}, x_{j}\right\rangle\right|\right\}^{\frac{1}{2 q}}
\end{array}\right.
$$

In particular, we have for $q=1$ that

$$
\|\mathbf{x}\|_{h, n, 1} \leq\left\{\begin{array}{l}
\left\{\sum_{i=1}^{n}\left\|x_{i}\right\|^{2}+\sum_{1 \leq i \neq j \leq n}\left|\left\langle x_{i}, x_{j}\right\rangle\right|\right\}^{\frac{1}{2}} \\
\left\{\left(\sum_{i=1}^{n}\left\|x_{i}\right\|^{2 r}\right)^{\frac{1}{r}}\right. \\
\left.+(n-1)^{\frac{1}{p}}\left(\sum_{1 \leq i \neq j \leq n}\left|\left\langle x_{i}, x_{j}\right\rangle\right|^{r}\right)^{\frac{1}{r}}\right\}^{\frac{1}{2}} \\
\text { where } p>1, \frac{1}{p}+\frac{1}{r}=1 \\
\left\{\max _{1 \leq i \leq n}\left\|x_{i}\right\|^{2}+(n-1) \max _{1 \leq i \neq j \leq n}\left|\left\langle x_{i}, x_{j}\right\rangle\right|\right\}^{\frac{1}{2}}
\end{array}\right.
$$

while for $q=2$ that

$$
\|\mathbf{x}\|_{h, e}^{2} \leq\left\{\begin{array}{l}
\max _{1 \leq i \leq n}\left\{\left\|x_{i}\right\|\right\}\left\{\sum_{i=1}^{n}\left\|x_{i}\right\|^{2}+\sum_{1 \leq i \neq j \leq n}\left|\left\langle x_{i}, x_{j}\right\rangle\right|\right\}^{\frac{1}{2}} \\
\|\mathbf{x}\|_{h, n, 2 p}\left\{\left(\sum_{i=1}^{n}\left\|x_{i}\right\|^{2 r}\right)^{\frac{1}{r}}\right. \\
\left.+(n-1)^{\frac{1}{p}}\left(\sum_{1 \leq i \neq j \leq n}\left|\left\langle x_{i}, x_{j}\right\rangle\right|^{r}\right)^{\frac{1}{r}}\right\}^{\frac{1}{2}} \\
\text { where } p>1, \frac{1}{p}+\frac{1}{r}=1 \\
\|\mathbf{x}\|_{h, e}\left\{\max _{1 \leq i \leq n}\left\|x_{i}\right\|^{2}+(n-1) \max _{1 \leq i \neq j \leq n}\left|\left\langle x_{i}, x_{j}\right\rangle\right|\right\}^{\frac{1}{2}}
\end{array}\right.
$$


Proof. If we take in (4.12) $c_{i}=\overline{\left\langle x_{i}, y\right\rangle}\left|\left\langle x_{i}, y\right\rangle\right|^{q-2}, i \in\{1, \ldots, n\}$, then we get

$$
\begin{aligned}
& \left(\sum_{i=1}^{n}\left|\left\langle x_{i}, y\right\rangle\right|^{q}\right)^{2} \\
& \leq\|y\|^{2} \times\left\{\begin{array}{l}
\max _{1 \leq i \leq n}\left\{\left|\left\langle x_{i}, y\right\rangle\right|^{2(q-1)}\right\}\left\{\sum_{i=1}^{n}\left\|x_{i}\right\|^{2}+\sum_{1 \leq i \neq j \leq n}\left|\left\langle x_{i}, x_{j}\right\rangle\right|\right\} ; \\
\left(\sum_{i=1}^{n}\left|\left\langle x_{i}, y\right\rangle\right|^{2 p(q-1)}\right)^{\frac{1}{p}}\left\{\left(\sum_{i=1}^{n}\left\|x_{i}\right\|^{2 r}\right)^{\frac{1}{r}}\right. \\
\left.+(n-1)^{\frac{1}{p}}\left(\sum_{1 \leq i \neq j \leq n}\left|\left\langle x_{i}, x_{j}\right\rangle\right|^{r}\right)^{\frac{1}{r}}\right\}, \\
\text { where } p>1, \frac{1}{p}+\frac{1}{r}=1 ; \\
\sum_{i=1}^{n}\left|\left\langle x_{i}, y\right\rangle\right|^{2(q-1)}\left\{\max _{1 \leq i \leq n}\left\|x_{i}\right\|^{2}+(n-1) \max _{1 \leq i \neq j \leq n}\left|\left\langle x_{i}, x_{j}\right\rangle\right|\right\},
\end{array}\right.
\end{aligned}
$$

for any $\mathbf{x} \in H^{n}$ with $\mathbf{x}=\left(x_{1}, \ldots, x_{n}\right)$ and $y \in H$.

If we take the square root in this inequality, then we get

$$
\sum_{i=1}^{n}\left|\left\langle x_{i}, y\right\rangle\right|^{q}
$$

$$
\leq\|y\| \times\left\{\begin{array}{l}
\max _{1 \leq i \leq n}\left\{\left|\left\langle x_{i}, y\right\rangle\right|^{q-1}\right\}\left\{\sum_{i=1}^{n}\left\|x_{i}\right\|^{2}+\sum_{1 \leq i \neq j \leq n}\left|\left\langle x_{i}, x_{j}\right\rangle\right|\right\}^{1 / 2} \\
\left(\sum_{i=1}^{n}\left|\left\langle x_{i}, y\right\rangle\right|^{2 p(q-1)}\right)^{\frac{1}{2 p}}\left\{\left(\sum_{i=1}^{n}\left\|x_{i}\right\|^{2 r}\right)^{\frac{1}{r}}\right. \\
\left.+(n-1)^{\frac{1}{p}}\left(\sum_{1 \leq i \neq j \leq n}\left|\left\langle x_{i}, x_{j}\right\rangle\right|^{r}\right)^{\frac{1}{r}}\right\}^{1 / 2}, \\
\text { where } p>1, \frac{1}{p}+\frac{1}{r}=1 ; \\
\left(\sum_{i=1}^{n}\left|\left\langle x_{i}, y\right\rangle\right|^{2(q-1)}\right)^{1 / 2}\left\{\max _{1 \leq i \leq n}\left\|x_{i}\right\|^{2}+(n-1) \max _{1 \leq i \neq j \leq n}\left|\left\langle x_{i}, x_{j}\right\rangle\right|\right\}^{1 / 2}
\end{array}\right.
$$

for any $\mathbf{x} \in H^{n}$ with $\mathbf{x}=\left(x_{1}, \ldots, x_{n}\right)$ and $y \in H$.

If we take the supremum over $y \in H,\|y\| \leq 1$, then we get

$$
\|\mathbf{x}\|_{h, n, q}^{q} \leq\left\{\begin{array}{l}
\max _{1 \leq i \leq n}\left\{\left\|x_{i}\right\|^{q-1}\right\}\left\{\sum_{i=1}^{n}\left\|x_{i}\right\|^{2}+\sum_{1 \leq i \neq j \leq n}\left|\left\langle x_{i}, x_{j}\right\rangle\right|\right\}^{1 / 2} ; \\
\|\mathbf{x}\|_{h, n, 2 p(q-1)}^{q-1}\left\{\left(\sum_{i=1}^{n}\left\|x_{i}\right\|^{2 r}\right)^{\frac{1}{r}}\right. \\
\left.+(n-1)^{\frac{1}{p}}\left(\sum_{1 \leq i \neq j \leq n}\left|\left\langle x_{i}, x_{j}\right\rangle\right|^{r}\right)^{\frac{1}{r}}\right\}^{1 / 2}, \\
\text { where } p>1, \frac{1}{p}+\frac{1}{r}=1 ; \\
\|\mathbf{x}\|_{h, n, 2(q-1)}^{q-1}\left\{\max _{1 \leq i \leq n}\left\|x_{i}\right\|^{2}+(n-1) \max _{1 \leq i \neq j \leq n}\left|\left\langle x_{i}, x_{j}\right\rangle\right|\right\}^{1 / 2},
\end{array}\right.
$$

for any $\mathbf{x} \in H^{n}$ with $\mathbf{x}=\left(x_{1}, \ldots, x_{n}\right)$, which produces the desired result (4.13). 
The interested reader may obtain other similar results by utilising the following inequalities obtained in [11]

Lemma 10. Let $y, x_{1}, \ldots, x_{n}$ be vectors of an inner product space $(H ;\langle\cdot, \cdot\rangle)$ and $c_{1}, \ldots, c_{n} \in \mathbb{K}$. Then one has the inequalities:

$$
\left|\sum_{i=1}^{n} c_{i}\left\langle x_{i}, y\right\rangle\right|^{2} \leq\|y\|^{2} \times\left\{\begin{array}{l}
D \\
E \\
F
\end{array},\right.
$$

where

$$
\begin{aligned}
& D:=\left\{\begin{array}{l}
\max _{1 \leq k \leq n}\left|c_{k}\right|^{2} \sum_{i, j=1}^{n}\left|\left\langle x_{i}, x_{j}\right\rangle\right| \\
\max _{1 \leq k \leq n}\left|c_{k}\right|\left(\sum_{i=1}^{n}\left|c_{i}\right|^{r}\right)^{\frac{1}{r}}\left[\sum_{i=1}^{n}\left(\sum_{j=1}^{n}\left|\left\langle x_{i}, x_{j}\right\rangle\right|\right)^{s}\right]^{\frac{1}{s}}, \quad r>1, \frac{1}{r}+\frac{1}{s}=1 \\
\max _{1 \leq k \leq n}\left|c_{k}\right| \sum_{k=1}^{n}\left|c_{k}\right| \max _{1 \leq i \leq n}\left(\sum_{j=1}^{n}\left|\left\langle x_{i}, x_{j}\right\rangle\right|\right)
\end{array}\right. \\
& E:= \begin{cases}\left(\sum_{k=1}^{n}\left|c_{k}\right|^{p}\right)^{\frac{1}{p}} \max _{1 \leq i \leq n}\left|c_{i}\right|\left(\sum_{i=1}^{n}\left(\sum_{j=1}^{n} \mid\left\langle x_{i}, x_{j}\right\rangle\right)^{q}\right)^{\frac{1}{q}}, & p>1, \frac{1}{p}+\frac{1}{q}=1 ; \\
\left(\sum_{k=1}^{n}\left|c_{k}\right|^{p}\right)^{\frac{1}{p}}\left(\sum_{i=1}^{n}\left|c_{i}\right|^{t}\right)^{\frac{1}{t}}\left[\sum_{i=1}^{n}\left(\sum_{j=1}^{n}\left|\left\langle x_{i}, x_{j}\right\rangle\right|^{q}\right)^{\frac{u}{q}}\right]^{\frac{1}{u}}, & p>1, \frac{1}{p}+\frac{1}{q}=1 ; \\
& t>1, \frac{1}{t}+\frac{1}{u}=1 ; \\
\left(\sum_{k=1}^{n}\left|c_{k}\right|^{p}\right)^{\frac{1}{p}} \sum_{i=1}^{n}\left|c_{i}\right| \max _{1 \leq i \leq n}\left\{\left(\sum_{j=1}^{n}\left|\left\langle x_{i}, x_{j}\right\rangle\right|^{q}\right)^{\frac{1}{q}}\right\}, & p>1, \frac{1}{p}+\frac{1}{q}=1 ;\end{cases}
\end{aligned}
$$

and

$$
F:=\left\{\begin{array}{c}
\left.\sum_{k=1}^{n}\left|c_{k}\right| \max _{1 \leq i \leq n}\left|c_{i}\right| \sum_{i=1}^{n}\left[\max _{1 \leq j \leq n} \mid\left\langle x_{i}, x_{j}\right\rangle\right]\right] \\
\sum_{k=1}^{n}\left|c_{k}\right|\left(\sum_{i=1}^{n}\left|c_{i}\right|^{m}\right)^{\frac{1}{m}}\left(\sum_{i=1}^{n}\left[\max _{1 \leq j \leq n}\left|\left\langle x_{i}, x_{j}\right\rangle\right|\right]^{l}\right)^{\frac{1}{l}} \\
m>1, \frac{1}{m}+\frac{1}{l}=1 \\
\left(\sum_{k=1}^{n}\left|c_{k}\right|\right)^{2} \max _{i, 1 \leq j \leq n}\left|\left\langle x_{i}, x_{j}\right\rangle\right| .
\end{array}\right.
$$

\section{REFERENCES}

[1] R. Bellman, Almost orthogonal series, Bull. Amer. Math. Soc., 50 (1944), 517-519.

[2] M. Biernacki, H. Pidek and C. Ryll-Nardzewski, Sur une inégalité entre des intégrales définies. (French) Ann. Univ. Mariae Curie-Sktodowska. Sect. A. 4, (1950). 1-4.

[3] R. P. Boas, A general moment problem, Amer. J. Math., 63 (1941), 361-370.

[4] E. Bombieri, A note on the large sieve, Acta Arith., 18 (1971), 401-404. 
[5] S. S. Dragomir, Another Grüss type inequality for sequences of vectors in normed linear spaces and applications, J. Comp. Analysis \& Appl., 4(2) (2002), 157-172.

[6] S. S. Dragomir, A Grüss type inequality for sequences of vectors in normed linear spaces, Tamsui Oxf. J. Math. Sci., 20(2) (2004), 143-159.

[7] S. S. Dragomir, A counterpart of Schwarz's inequality in inner product spaces, East Asian Math. J., 20 (1) (2004), 1-10. Preprint RGMIA Res. Rep. Coll. 6 (2003), Supplement, Art. 18. [Online http://rgmia.org/papers/v6e/CSIIPS.pdf].

[8] S. S. Dragomir, A survey on Cauchy-Bunyakovsky-Schwarz type discrete inequalities. J. Inequal. Pure Appl. Math. 4 (2003), no. 3, Article 63, 142 pp. [Online https://www .emis. de/journals/JIPAM/article301.html?sid=301] .

[9] S. S. Dragomir, The hypo-Euclidean norm of an $n$-tuple of vectors in inner product spaces and applications. J. Inequal. Pure Appl. Math. 8 (2007), No. 2, Article 52, 22 pp. [Online https://www .emis. de/journals/JIPAM/article854.html?sid=854] .

[10] S. S. Dragomir, On the Boas-Bellman inequality in inner product spaces. Bull. Austral. Math. Soc. 69 (2004), no. 2, 217-225.

[11] S. S. Dragomir, On the Bombieri inequality in inner product spaces. Libertas Math. 25 (2005), $13-26$.

[12] S. S. Dragomir and G. L. Booth, On a Grüss-Lupaş type inequality and its applications for the estimation of $p$-moments of guessing mappings, Math. Comm., 5 (2000), 117-126.

[13] H. Heilbronn, On the averages of some arithmetical functions of two variables, Mathematica, 5(1958), 1-7.

[14] D. S. Mitrinović ITRINOVIĆ, J. E. Pečarić and A. M. Fink, Classical and New Inequalities in Analysis, Kluwer Academic Publishers, 1993.

[15] J. E. Pečarić, On some classical inequalities in unitary spaces, Mat. Bilten (Scopje), 16(1992), 63-72.

[16] A. Sheikhhosseini, M. S. Moslehian and K. Shebrawi, Inequalities for generalized Euclidean operator radius via Young's inequality. J. Math. Anal. Appl. 445 (2017), no. 2, 1516-1529.

[17] O. Shisha and B. Mond, Bounds on differences of means, Inequalities, Academic Press Inc., New York, 1967, 293-308.

${ }^{1}$ Mathematics, College of Engineering \& Science, Victoria University, PO Box 14428 , Melbourne City, MC 8001, Australia.

E-mail address: sever.dragomir@vu.edu.au

$U R L:$ http://rgmia.org/dragomir

${ }^{2}$ DSt-NRF Centre of Excellence, in the Mathematical and Statistical Sciences, School of Computer Science \& Applied Mathematics, University of the Witwatersrand, Private Bag 3, Johannesburg 2050, South Africa 